\title{
Study and Analysis of Energy Efficient Cluster based Routing Protocol
}

\author{
Abhishek Kumar Pandey \\ M.Tech. Scholar \\ Computer Science \& Engg. \\ K.N.I.T. Sultanpur (U.P) India
}

\author{
Samir Srivastava \\ Associate Professor \\ Computer Science \& Engg. \\ K.N.I.T. Sultanpur (U.P) India
}

\begin{abstract}
Constant development and improvement in wireless sensor network (WSN) technology has supplied some new opportunities of small land minor-cost sensor nodes with capacity of sensing various permuted physical and environmental conditions, data processing, and wireless communication. The outcome of diversity of sensing effectiveness is in the excess of application areas. But to an extent, the earliest form of wireless sensor networks require successful desired approach for data forwarding and processing. In WSN, the sensor nodes have a fixed transmission range, and their refining and storage potential as well as their energy systems are also bounded. Routing protocols for wireless sensor networks are the cause for maintaining the routes in the network and also settled some trust worthy multi-hop communication under certain circumstances. In this work the routing protocols are examined for Wireless Sensor Network and compare their attributes. The main important design points for a sensor network are maintenance of the energy available in each sensor node. Increasing the network lifetime has decisive importance in wireless sensor networks. Lots of routing algorithms have been developed in the process. Above all the algorithms, clustering algorithms reached most closely in improving the network life time and finally the efficiency of the nodes in it. Clustering provides an efficient way for increasing the lifetime of a wireless sensor Network. This work briefly compares four renowned routing protocols namely, LEACH, SEP, TEEN, and EAMMH for various general scenarios and full fledge analysis of the simulation results against known metrics with energy and network life time being the most important .In this research work the results and observations made from the analyses of results about these protocols are presented.
\end{abstract}

\section{Keywords}

Wireless sensor network LEACH, SEP, TEEN and EAMMH energy-efficient, network lifetime.

\section{INTRODUCTION}

Wireless Sensor Network (WSN) is a network of one or more sensors, wireless sensors deployed in a remote or hazard area to sense various types of physical information from the environment [1]. The information sensed by these sensors or nodes is then processed and has been sent to Base Station (BS) for assessment. The nodes are dense and monitoring of these nodes is very complex Particularly in the cases when the nodes are distributed in the regions where the physical interfere of the human being is not possible[2]. The network once established, keep on sensing the information and the energy of the nodes Keep on dissipating whenever, they receive some information and send it further to other nodes or BS.

\begin{abstract}
Many of routing protocols have been proposed to make nodes more energy efficient. Dense nature of this sensor create the situation when the redundant information is transferred to the base station, along with this the energy of the nodes is also dissipated. To overcome from these problems various clustering algorithms were proposed. The entire network of nodes is divided into a number of clusters; the data aggregation is performed within the cluster and then transmitted to the BS. Clustering helps in decreasing of redundancy and increasing the lifetime of the network [3]. The LEACH [4] and SEP [5] TEEN and EAMMH are such clustering protocols. The rest of the paper is organized as follows:
\end{abstract}

\section{METHODOLOGIES}

In this paper we reviewed and analyzed some modern energy efficient protocols [6] like LEACH, SEP, TEEN and EAMMH

LEACH (Low Energy Adaptive Clustering Hierarchy) Heinzelman et al. [7] who proposed a protocol which is based on the hierarchical routing known as LEACH. It is a clustering based protocol in which some clusters are formed and within the cluster the nodes will be chosen cluster head. The selection of cluster head is a random process and cluster heads are rotated within each round of data processing. Due to the cluster head randomization, cluster heads energy will be well distributed within the each node of the sensor network. It uses the localized coordination which enhances the scalability and fault tolerance. According to the author cluster also changes after some given time interval and hence it creates a uniform energy dissipation within the whole network. The whole operation of the leach protocol will be done in two steps: The Setup phase and The Steady State phase. Under the Setup phase clustering and random selection of cluster head by node within the cluster will be done. And in the steady state phase data transmission from node to cluster head and cluster head to base station will be done.

\section{Advantages of LEACH protocol are:}

1. It restricts most of the communication within the clusters, and therefore provides scalability in the network.

2. In LEACH there is a Single-hop routing from node to cluster head, thus energy is saved.

3. Leach Protocol does not need location information of the nodes to build the clusters. Therefore, it is powerful and simple

4. Leach is dynamic clustering and appropriate for applications where constant monitoring is required and data gathering occurs periodically to a centralized location. 


\section{Disadvantages of LEACH protocol are:}

1. Nodes have different energy level, but $\mathrm{CH}$ is selected obstructive.

2. The performance of LEACH protocol is not ideal for large network.

SEP (Stable Election Protocol) SEP [8] protocol is an enhancement of LEACH [5] protocol which uses clustering based routing strategy based on the node heterogeneity of the sensor node in the networks. In this protocol, few of the sensor nodes having high energy and this type of a sensor nodes is referred as the advanced nodes and the chance of the advanced nodes to become $\mathrm{CHs}$ is more as compared to the normal nodes and the normal nodes having low energy as compared to the advanced nodes in the network. Distributed method has been used as SEP [10] strategy to select a CH in WSNs. It is heterogeneity-aware protocol and selection of $\mathrm{CH}$ probabilities of nodes is weighted by initial energy of each node compared to the other nodes in WSN. Therefore, SEP protocol is based on two levels of node heterogeneity as normal nodes and advanced nodes.

1. Let, $m$ is the fraction of total number of nodes $n$, which are deployed with $\alpha$ times more energy than the others nodes.

Energy per normal node $=\mathrm{e}_{0}$

Number of advanced nodes $=\mathrm{m} \times \mathrm{n}$

Energy per advanced node $=\mathrm{e}_{0} \mathrm{x}(1+\alpha)$

2. These powerful nodes are as advanced nodes.

3. The remaining $(1-\mathrm{m}) \times \mathrm{n}$ nodes are as normal nodes.

4. Probability of normal nodes to become $\mathrm{CH}_{\mathrm{S}}$ is calculated as $\mathrm{P}_{\text {nor }}=\mathrm{p}_{\text {opt }} /(1+\mathrm{m} . \alpha)$

5. Probability of advanced nodes to become $\mathrm{CH}_{\mathrm{S}}$ is calculated as $\mathrm{P}_{\mathrm{adv}}=\mathrm{p}_{\mathrm{opt}}(1+\alpha) /(1+\mathrm{m} . \alpha)$

$\mathrm{P}_{\mathrm{opt}}$ is the optimal probability of each node to become $\mathrm{CH}$ in the network. In SEP [8][9] strategy, selection of $\mathrm{CH}$ is done randomly on probability basis for each node. Sensor nodes continuously sense data and transmit it to their associated $\mathrm{CH}$ and $\mathrm{CH}$ transmit that data it to the sink or base station (BS).This system can be further improved by increasing the value of $m$ or $\mathrm{p}_{\mathrm{adv}}$. Due to advance nodes this sensor network is known as heterogeneity, SEP [8] strategy results in high stable time period, high network lifetime and high throughput.

\section{Advantages of SEP protocol are:}

1. Any identification or global knowledge of energy of sensor node is not required in SEP [8] technique at each selection round of cluster head.

\section{Disadvantages of SEP protocol are:}

1. The cluster head $(\mathrm{CH})$ selection among sensor nodes are not dynamic, which results that nodes that are far away from the powerful nodes will die first.

TEEN (Threshold Sensitive Energy Efficient Sensor Network Protocol) these protocols TEEN [11] are used for the real time applications or for real time data packet. Real time data packets have some time limitation, data sending within certain time period. According to the TEEN protocol, The Sensor node senses the physical medium continuously, but sending of the data is less frequent. Cluster head sending a hard threshold to entire sensor node within the cluster, it is called the attribute for the sensed data. It will also send the soft threshold, which can change the sense attribute and also trigger the particular node to turn on its transmitter and data sending process will start. Hence hard threshold is used to make the less frequent data transmission of the sensed data and data transmission start when the sensed attribute is within the range of our interest. When the hard threshold getting no change then the number of the transmission by the soft threshold will be reduced. If the soft threshold is small then the accurate data transmission occurs and by this way the energy consumption of the whole network increases. So we see the some tradeoff between energy efficiency and accuracy.

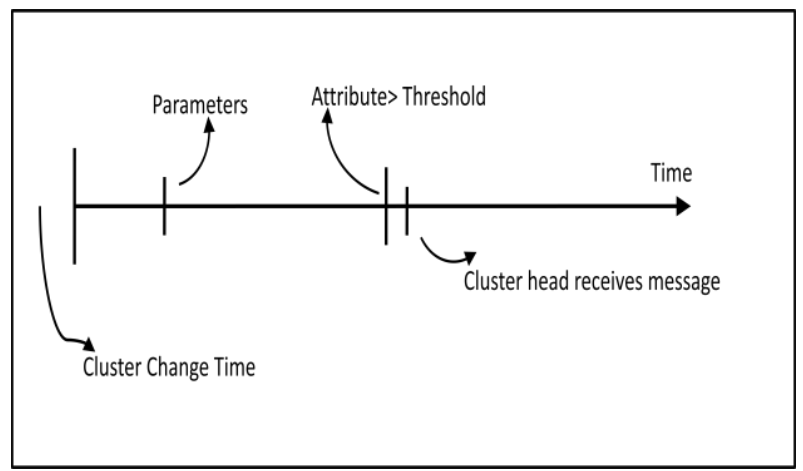

Fig.1: Operation of TEEN

The above figure 1 describes that when a cluster head is changed of a cluster then the new parametric quantity are broadcasted. This routing protocol have some drawback, the node will not communicate to other at the time when any thresholds will not received by the node. So due to this data will not be transmitted to the sink and we cannot receive any data from sensor network. The physical medium is sensed continuously by the sensor motes, after some time the parametric value of the set of attribute getting its hard threshold, and the transmitter turns on by the node and data sending will start. The sensed value is stored in to the internal variable [11]. The data node transmits the data to sensor node in only the current cluster period if it satisfies the two conditions.

1. The value of the hard threshold should be less than the present parametric value of the attribute which is sensed by the sensor node.

2. The value stored in the internal variable should from differ from the parametric value of the sensed attribute. This difference should be greater than or equal to the soft threshold.

Advantages of TEEN protocol are:

1. TEEN [12] is suitable for the time critical applications.

2. TEEN protocol is relatively efficient. In terms of energy consumption and response time

3. According to the criteria of TEEN protocol, soft threshold [13] value can be varied.

4. smaller value of soft threshold produces more accurate result of the WSN.

Disadvantages of TEEN protocol are:

1. In the TEEN protocol if the threshold value is not reached the sensor nodes will never communicate, No data will be achieved from the sensor network 
at all and it will be unknown even if all the sensor nodes die.

2. Cluster heads (CHs) will always wait for data from their nodes and keep their transmitter on.

\section{EAMMH (Energy aware Multi-Hop Multi-Path}

Hierarchy) the main goal of LEACH protocol is to decrease the energy consumption or, to increase the network lifetime. To make this happen many ideas are proposed for $\mathrm{CH}$ selection but they were based on mainly the node's (to be selected as $\mathrm{CH}$ ) energy level. [14]The node having larger energy level will be selected as $\mathrm{CH}$ most of the times. But here in the new proposed scheme not only the node's energy level is considered but also its location or position both within the $\mathrm{CH} \&$ from outside the cluster(neighbor clusters) are considered. We know that there may a number of nodes in a cluster \& there is always a $\mathrm{CH}$. for example, if the $\mathrm{CH}$ lies at a far-away position from the mass of nodes. So to communicate between $\mathrm{CH} \&$ sensor nodes, since the distance between them is high, energy consumption for the communication is also high. That means, the higher the distance between $\mathrm{CH} \&$ sensor nodes the greater the energy consumption. Here a new idea to select the $\mathrm{CH}$ is given below:

1. Selection of the $\mathrm{CH}$ in the dense node zone. : For example, you are announcing something. If the persons, for whom your announcement is, are very far from you, you have to shout more to make them listen to it but if those persons are close to you, you won't have to shout that much. That means, if nodes are close to the $\mathrm{CH}$, then consumption of energy is minimum.

2. Assume a cluster is surrounded by 7 clusters. So $7 \mathrm{CH}$ can communicate with the central $\mathrm{CH}$. This central $\mathrm{CH}$ should be at an optimal distance from those $\mathrm{CH}$. That means the distance between them should be balanced or on average.

Say, C0,C1,C2,C3,C4,C5,C6,C7 are the $\mathrm{CH}$ of cluster 0 (central cluster), cluster 1 , cluster 2 , cluster 3 , cluster 4 , cluster 5 , cluster 6 , cluster 7 respectively. There should not be a huge difference among distances between $\mathrm{C} 0-\mathrm{C} 1, \mathrm{C} 0-\mathrm{C} 2$, $\mathrm{C} 0-\mathrm{C} 3, \mathrm{C} 0-\mathrm{C} 4, \mathrm{C} 0-\mathrm{C} 5, \mathrm{C} 0-\mathrm{C} 6$ and $\mathrm{C} 0-\mathrm{C} 7$.

Hence, energy consumption will be in control.

$(\mathrm{n})=\frac{p}{1-p\left(r \bmod \frac{1}{p}\right)}\left(\frac{E(i) \cdot E}{E \max }\right)\left(\frac{D a v g}{\sum D \text { inter_node }}\right) \mathrm{n} \in \mathrm{G} \forall$

$\mathrm{n} \operatorname{not} \epsilon \mathrm{G} \forall T n=0$

Where $S$ (i).E is the current energy of each node and $E_{\text {max }}$ is the initial energy of each node. $\mathrm{D}_{\text {avg }}$ is the average distance from all other nodes in the cluster. $\mathrm{D}_{\text {inter_nodes }}$ is the distance between any two nodes in the cluster. Here with the original formula two factors are multiplied.

(1) Average distance from other nodes in same cluster/ $\sum$ inter -node distance.

This factor checks whether the node, to be selected as $\mathrm{CH}$, belongs to a density popular area as well as the distance from the node to the other nodes within the cluster is on average.

(2) Current energy of the node/Initial energy of each node.

This factor suggests that each node computes the percentage of its own energy level and the aggregate energy remain in the network. With this value each node decides if it becomes cluster-head for this round or not. High energy nodes will more likely to become cluster-heads than low-energy nodes.

The operation of the EAMMH protocol is broken into rounds where starting of each round with a set -up phase, when the clusters are prepared, followed by a steady- state phase, when data transfers to the base station occur. Initially the user has to give the input which is in the form of number of nodes. Once the nodes are deployed, every node uses the neighbor discovery algorithm to discover its neighbor nodes. Using the cluster head selection algorithm cluster heads are selected between the nodes. These cluster heads broadcasts the advertisement message to all its neighboring nodes and thus clusters are formed with a fixed bound size.

Each node in the cluster maintaining the routing table in which routing information of the nodes are update.

DRAND (distributed randomized time slot assignment algorithm) [15] method is used; it allows number of nodes to allocate the same frequency channel by dividing the signal into different time slots. The cluster head aggregates the data from all the nodes in the cluster and this aggregated data is transmitted to the base station.

\section{Setup Phase}

Initially, after the node deployment the neighbor discovery takes place. This can be done using many methods like: $k$ of- $n$ approach, ping, beacon messaging. After the neighbor discovery, when cluster are being created, each node decides whether or not to become a cluster-head for the current round. This decision method is similar to the one used in LEACH. The setup phase operates in the following sequence:

\section{1. $\mathrm{CH}$ (Cluster Head) Selection}

\section{Cluster Formation}

\section{Data Transmission Phase}

Once the clusters are created, the sensor nodes are allotted timeslots to send the data. Assuming nodes always have data to send, they transmit it at their allotted time interval. When a node receives data from one its neighbors, it aggregates it with its own data. While forwarding the aggregated data, it has to choose an optimal path from its routing table entries. It uses a heuristic function to make this decision and the heuristic function is given by,

$\mathrm{h}=\mathrm{K}\left(\mathrm{E}_{\mathrm{avg}} / \mathrm{h} * \mathrm{t}\right)$

Where $\mathrm{K}$ is a constant, Eavg is the average energy of the current path, $\mathrm{h}_{\min }$ is minimum hop count in current path, $\mathrm{t}=$ traffic in the current path. The path with highest heuristic value is chosen. If this path ${ }^{\text {ee }} \mathrm{E}_{\min }>$ threshold, it is chosen.

Else the path with the next highest heuristic value is chosen, where $\mathrm{E}_{\mathrm{min}}=\mathrm{E} /$ const

The constant may be any integer value like 10 .

If no node in the routing table has $\mathrm{E}_{\min }$ greater than threshold energy, it picks the node with highest minimum energy. The information about the paths and routing table entries at each node becomes stale after a little while. The Min heuristic values calculated based on the stale information often leads to wrong decisions. Hence the nodes are to be supplied with fresh information periodically. This will increase the accuracy and timeliness of the heuristic function. During the operation of each round, the necessary information is exchanged at regular intervals. The interval of periodic updates is chosen wisely such that the node does not base its decisions on the 
stale information and at the same time, the periodic update does not overload the network operation.

\section{SIMULATION AND ANALYSIS OF RESULTS}

We used MATLAB as a simulator for our implementation and performance evaluation of estimating simulations is to compare the performance of LEACH, SEP, TEEN and EAMMH protocols on the basis of energy consumption, lifetime of the sensor network

Performance attributes used in our MATLAB simulations are as follows:

1. Number of dead nodes during each round. (With variation of number of nodes)

2. Number of alive nodes during each round. (With variation of number of nodes)

3. Average energy of each node during each round. (With variation of number of nodes

\section{Network Settings}

The various parameter values taken for experiments are shown in the following table.

Table1. Initial Parameter Setting

\begin{tabular}{|l|l|}
\hline Parameter & Value \\
\hline Network size & $100 * 100$ \\
\hline Base Station Location & $(150,50)$ \\
\hline $\mathrm{E}_{0}$ & $0.3 \mathrm{~J}$ \\
\hline $\mathrm{P}_{\text {opt }}$ & 0.2 \\
\hline $\mathrm{n}$ & 200 \\
\hline$\alpha$ & 1 \\
\hline $\mathrm{m}$ & 0.2 \\
\hline $\mathrm{rmax}$ & 1000 \\
\hline
\end{tabular}

\subsection{Implementation Results of Protocols for $n=200$ nodes}

Initially we are keeping in mind that WSN consists of 200 sensor nodes, all sensor nodes are placed randomly in a region. For MATLAB simulation, we initialized some parameters like $\mathrm{E}_{\text {initialas }} 0.30$ Joule, $\mathrm{P}_{\text {opt }} 0.2, \alpha$ is 1 , no. of nodes is $200, \mathrm{~m}$ as 0.20 , and $\mathrm{E}_{0}$ is 0.30 Joule.

\subsubsection{Number of dead node during each round}

This section shows the results of different approaches and compares the quality of enhanced

Images. Fig. 2 plots the graph of nodes dead during each round. In Fig.2, LEACH protocol is shown as the blue curve, SEP protocol is shown as the green curve TEEN protocol is shown as the red curve and EAMMH protocol is shown as the cyan curve. As shown in the Fig. TEEN protocol has better performance as sensor nodes dies later and less as compared to LEACH, EAMMH and SEP protocol.

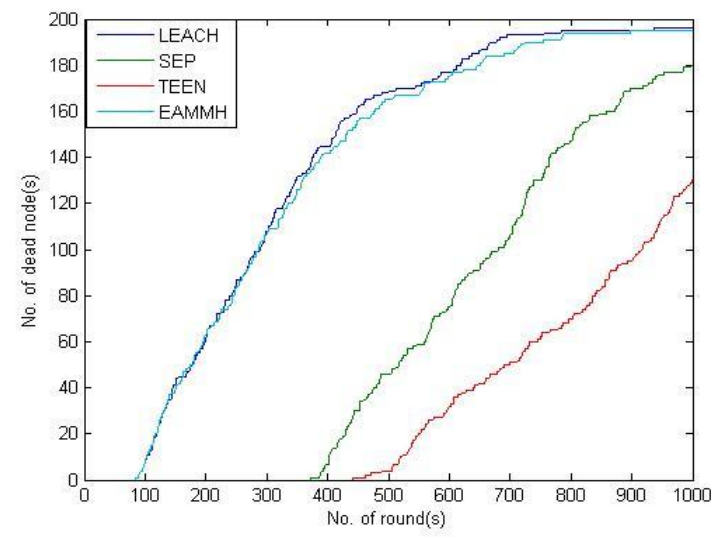

Fig.2: Nodes dead during each round

\subsubsection{Number of Alive node during each round}

In Fig.3, same colored curves have been used as in Fig. 2 for LEACH, SEP, TEEN and EAMMH. No. of nodes alive during each round is shown in Fig. 3 is the opposite of the graph of nodes dead during each round. Again TEEN protocol performs better as compared to LEACH, EAMMH and SEP protocol as shown in the graph. The graph plotted for nodes alive during each round shown in Fig.3

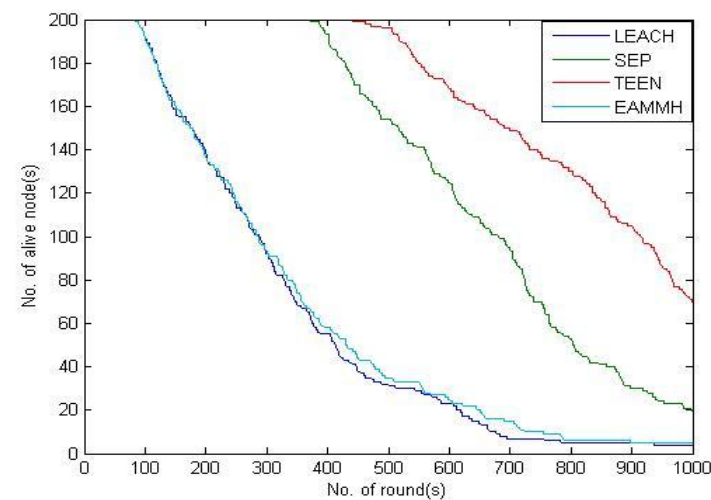

Fig.3: Nodes alive during each round

TEEN Protocol performs better as compare to LEACH, EAMMH and SEP because fewer nodes die or more nodes alive after each rounds as compared to LEACH, EAMMH and SEP protocols.

\subsubsection{Average energy of each node during each round}

This section shows the results of average energy of each node during each round. Fig. $4 a, 4 b, 4 c$ and $4 d$ plot the graph of average energy of each node during each round of $\mathrm{LEACH}$, SEP, TEEN and EAMMH protocol respectively. Fig. 4a of LEACH protocol shows parabolic nature which represents that average energy of each node decreases rapidly as the number of round increases.

Fig. 4b of SEP protocol shows linear nature at starting and at the end it shows a little parabolic nature. fig. $4 c$ of TEEN protocol shows almost linear nature from starting to end and a few energy remains after completion of all rounds. Whereas Fig. 4d of EAMMH protocol little perform better than LEACH protocol. Hence, TEEN protocol is more average energy efficient than LEACH, EAMMH and SEP protocol. 


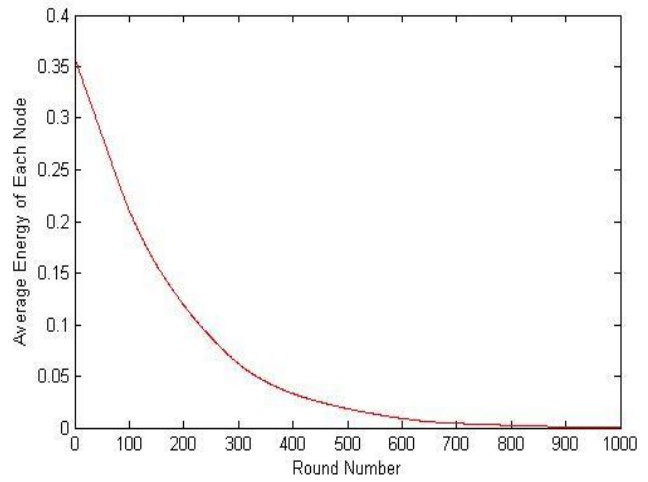

Fig: 4a (LEACH)

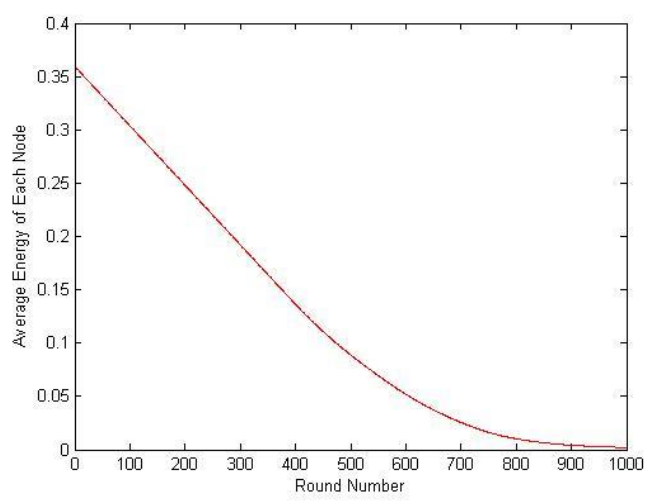

Fig: 4b (SEP)

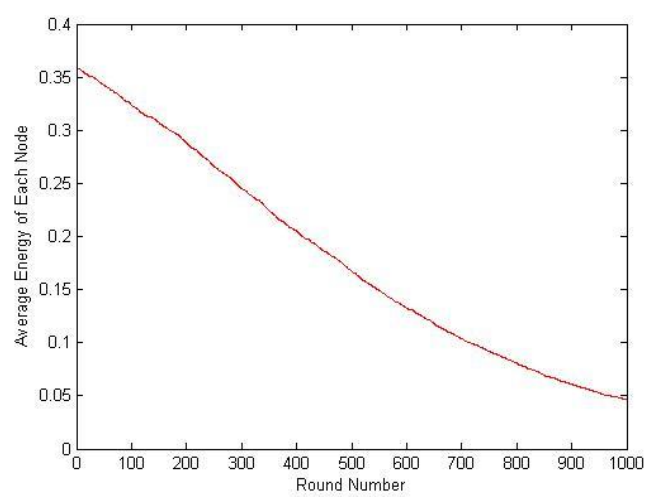

Fig: 4c (TEEN)

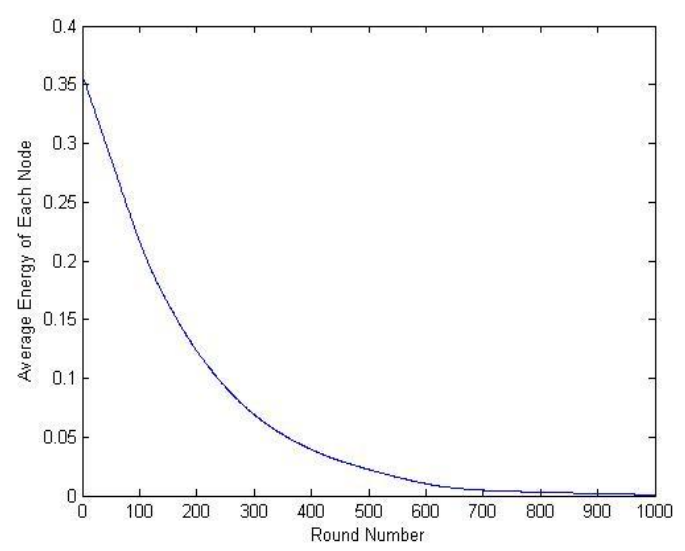

Fig: 4d (EAMMH)
Fig.4: Average energy of each node during each round

\subsection{Implementation Results of Protocols for $\mathrm{n}=100$ nodes}

We changed the parameters setting to different values. For MATLAB simulation, we initialized some parameters like $\mathrm{E}_{\text {initialas }} 0.30$ Joule, $\mathrm{P}_{\text {opt }} 0.2, \alpha$ is 1 , no. of nodes is $100, \mathrm{~m}$ as 0.20 , and $\mathrm{E}_{0}$ is 0.30 Joule.

\subsubsection{Number of dead node during each round}

As shown in the Fig.5 plots the graph of nodes dead during each round. LEACH protocol is shown as the blue curve, SEP protocol is shown as the green curve, TEEN protocol is shown as the red curve and EAMMH protocol is shown as the cyan curve. Again TEEN protocol has better performance as sensor nodes dies later and less as compared to LEACH, EAMMH and SEP protocol

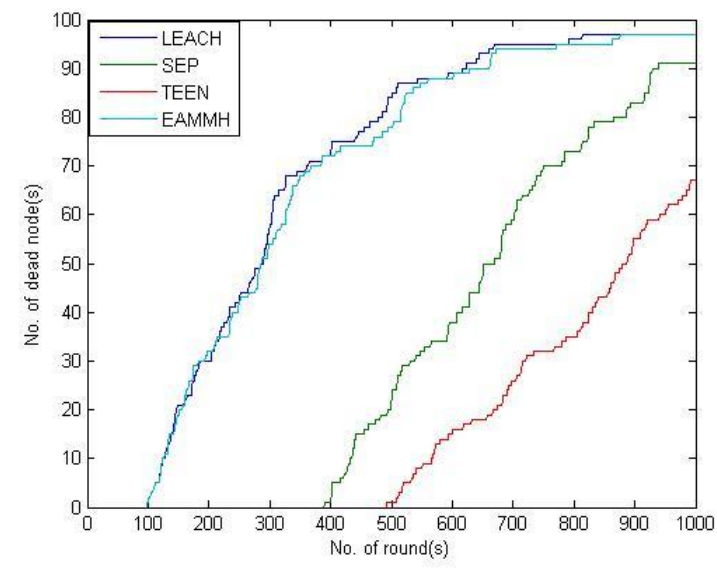

Fig.5: Nodes dead during each round

3.2.2 Number of Alive node during each round No. of nodes alive during each round is shown in Fig. 6 is the opposite of the graph of nodes dead during each round. Again TEEN protocol performs better as compared to LEACH, EAMMH and SEP protocol as shown in the graph. The graph plotted for nodes alive during each round shown in Fig 6.

TEEN Protocol performs better as compare to LEACH, EAMMH and SEP because fewer nodes die or more nodes alive after each rounds as compared to LEACH, EAMMH and SEP protocols.

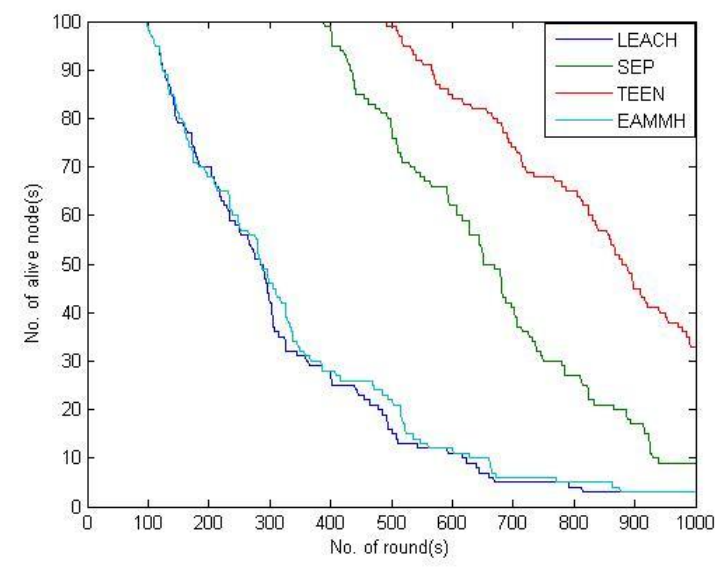

Fig.6: Nodes alive during each round 


\subsubsection{Average energy of each node during each round}

This section shows the results of average energy of each node during each round. Fig.7a, $7 \mathrm{~b}, 7 \mathrm{c}$ and $7 \mathrm{~d}$ plot the graph of average energy of each node during each round of LEACH, SEP, TEEN and EAMMH protocol respectively. Fig.7a of LEACH protocol shows parabolic nature which represents that average energy of each node decreases rapidly as the number of round increases.

Fig.7b of SEP protocol shows linear nature at starting and at the end it shows a little parabolic nature. Fig.7c of TEEN protocol shows almost linear nature from starting to end. Whereas Fig.7d of EAMMH protocol similar to LEACH protocol. But for small network SEP and TEEN shows same nature at the end. Hence, SEP and TEEN both protocol are almost similar in performance for small network than LEACH protocol

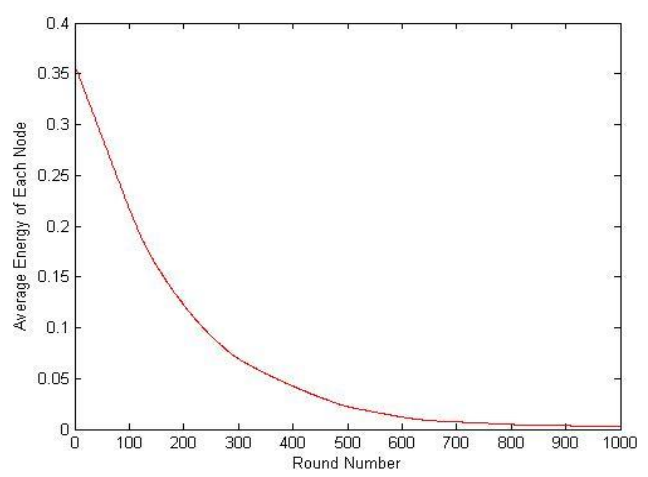

Fig: 7a (LEACH)

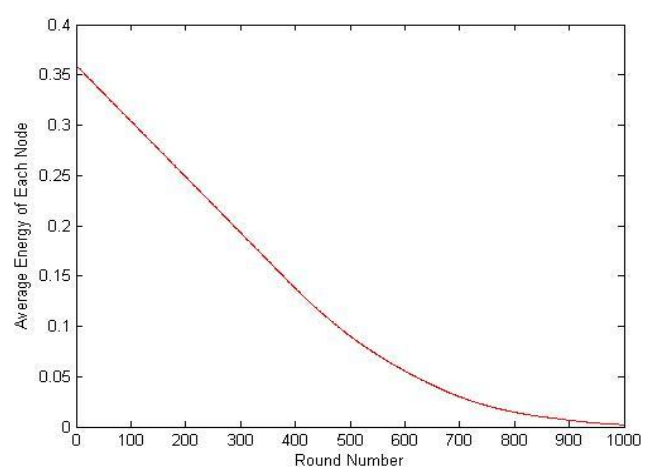

Fig: 7b (SEP)

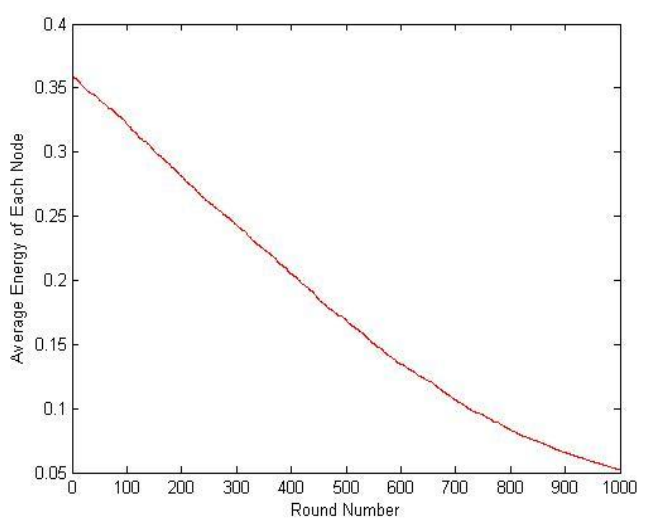

Fig: 7c (TEEN)

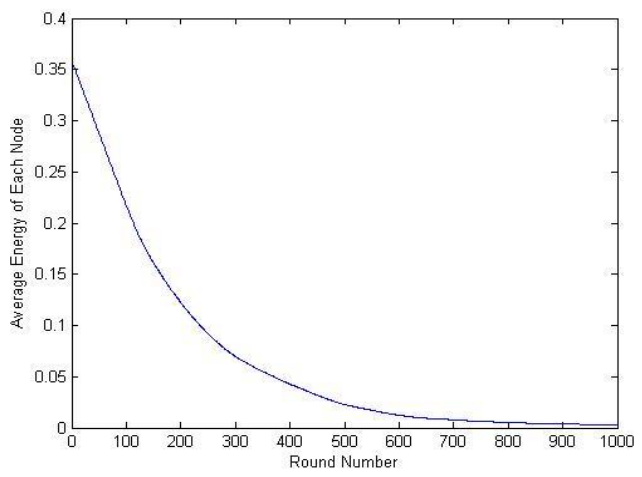

Fig: 7d (EAMMH)

Fig.7: Average energy of each node during each round

4. CONCLUSION AND FUTURE WORK

In this paper we have given the comparison of the four routing protocols; LEACH, SEP, TEEN and EAMMH respectively. By this comparison we identify the lifetime of the sensor network and energy consumption in different routing techniques. The results say that the life time of the sensor network using TEEN protocol is $33.67 \%$ better than LEACH; EAMMH protocol is $0.51 \%$ better than Leach and where as SEP is $7.65 \%$ better than LEACH. So the overall TEEN is best routing protocol in respect to life time and energy consumption of the sensor network. In future improvement of lifetime of the network and scalability of the node.

\section{REFERENCES}

[1] F. Akyidiz, Y. Sankarasubramaniam W. Su, andE. Cayirci, "A survey on sensor networks", IEEE Commun, August 2002

[2] Abishek Pandey, R.C. Tripathi, "A survey on wireless sensor network security", International Journal of Computer Applications (0975 - 8887) Volume 3 - No.2, PP-43-49, June 2010,

[3] J. N. Al-Karaki and A. E. Kamal, "Routing techniques in wireless sensor networks: a survey," IEEE Wireless Communications, vol. 11, no. 6,pp. 6-28,Dec. 2004.

[4] W. R. Heinzelman, A. Chandrakasan, and H. Balakrishnan."Energy-efficient communication protocol for wireless micro sensor networks", Proceedings of the 33rd Hawaii International Conference on System Sciences (HICSS-33), January 2000.

[5] G. Smaragdakis, I. Matta, A. Bestavros, "SEP: A Stable Election Protocol for clustered heterogeneous wireless sensor networks", Second International Workshop on Sensor and Actor Network Protocols and Applications (SANPA2004), 2004

[6] Johnson, M., Healy, M., van de Ven, P., Hayes, M.J., Nelson, J., Newe, T., Lewis, E., "A comparative review of wireless sensor network mote technologies", IEEE Sensors 2009, pp. 1439-1442.

[7] Ningbo Wang, HaoZhu, "An Energy Efficient Algorithm Based on LEACH Protocol", International Conference on Computer Science and Electronics Engineering (ICCSEE), 2012, pp. 339-342.

[8] Isla m, M M, Matin, M A, Mondol, T K, "Extended Stable Election Protocol (SEP) for three-level 
hierarchical clustered heterogeneous WSN", IET Conference on Wireless Sensor Syste ms 2012, pp. 1-4.

[9] O. Rehman, N. Javaid, B. Manzoor, A. Hafeez, A. Iqbal, M. Ishfaq, " Energy Consumption Rate based Stable Election Protocol (ECRSEP) for WSNs", Procedia Computer Science, Volume 19, 2013, pp. 932-937.

[10] Raju Pal, RituSindhu, Ajay K Sharma, "SEP-E (RCH): Enhanced Stable Election Protocol Based on Redundant Cluster Head Selection for HWSNs", 9th International Conference on Quality, Reliability, Security and Robustness in Heterogeneous Networks, 2013, Vol. 115, pp. 104-114.

[11] A. Manjeshwar and D.P. Agarwal, "TEEN: a routing protocol for enhanced efficiency in wireless sensor networks," 1st Int. Workshop Parallel Distributed Computing Issues Wireless Networks Mobile Computing, April 2001
[12] AratiManjeshwar, Agrawal, D.P., "TEEN: a routing protocol for enhanced efficiency in wireless sensor net works", Proceedings 15th International conference on Parallel and Distributed Processing Symposium 2001, pp. $2009-2015$.

[13] Aliouat, Z, Harous, S., "An efficient clustering protocol increasing wireless sensor net works life time", International Conference on Innovations in Information Technology (IIT), 2012, pp.194 - 199

[14] Abhishek Kumar Pandey and Samir Srivastava., "Survey on wireless sensor routing protocols", International journal of science and research, Volume 5, Issue 5 2016, pp. $1145-1149$

[15] Yu-quan, Zhang, and Wei Le i. "Improving the LEACH protocol for wireless sensor networks." (2010): 355 -359 